\title{
Identification of polymorphic loci in the deiodinase 2 gene and their associations with head dimensions in geese
}

\author{
Yan Deng ${ }^{1}$, Qian $\mathrm{Hu}^{1}$, Bincheng Tang ${ }^{1}$, Qingyuan Ouyang ${ }^{1}$, Shenqiang $\mathrm{Hu}^{1}$, Bo $\mathrm{Hu}^{1}$, \\ Jiwei $\mathrm{Hu}^{1}$, Hua He${ }^{1}$, Guohong Chen ${ }^{2}$, and Jiwen Wang ${ }^{1, *}$
}

\author{
* Corresponding Author: Jiwen Wang \\ Tel: +86-13608269159, Fax: +86-8352891889 \\ E-mail:wjw2886166@163.com \\ ${ }^{1}$ Farm Animal Genetic Resources Exploration \\ and Innovation Key Laboratory of Sichuan \\ Province, Sichuan Agricultural University, \\ Chengdu 611130, Sichuan, China \\ ${ }^{2}$ Key Laboratory of Animal Genetics and \\ Breeding and Molecular Design of Jiangsu \\ Province, Yangzhou University, Yangzhou \\ 225000, Jiangsu, China \\ ORCID \\ Yan Deng \\ https://orcid.org/0000-0002-3185-3810 \\ Qian $\mathrm{Hu}$ \\ https://orcid.org/0000-0002-7321-390X \\ Bincheng Tang \\ https://orcid.org/0000-0003-0248-4689 \\ Qingyuan Ouyang \\ https://orcid.org/0000-0003-4121-8770 \\ Shenqiang $\mathrm{Hu}$ \\ https://orcid.org/0000-0002-9256-8351 \\ $\mathrm{Bo} \mathrm{Hu}$ \\ https://orcid.org/0000-0003-0976-9070 \\ Jiwei Hu \\ https://orcid.org/0000-0002-4069-8875 \\ $\mathrm{Hua} \mathrm{He}$ \\ https://orcid.org/0000-0002-5131-3854 \\ Guohong Chen \\ https://orcid.org/0000-0002-4829-6504 \\ Jiwen Wang \\ https://orcid.org/0000-0001-8568-7654
}

Submitted Aug 24, 2021; Revised Sept 15, 2021 Accepted Oct 14, 2021
Objective: This study was conducted to clone and compare the molecular characteristics of the deiodinase 2 (DIO2) gene between Sichuan White geese and Landes geese, and to analyze the association between polymorphisms of the DIO2 gene and head dimensions in Tianfu meat geese.

Methods: The coding sequence of the DIO2 gene was cloned by polymerase chain reaction and vector ligation and aligned by DNAMAN software. A total of 350 Tianfu meat geese were used to genotype the polymorphisms of the DIO2 gene and measure the head dimensions. Association analysis between the polymorphisms of the DIO2 gene and head dimensions was carried out.

Results: An 840-bp coding sequence of the DIO2 gene was obtained and comparison analysis identified four polymorphic loci between Sichuan White geese and Landes geese. Further analysis showed that the dominant alleles for the four polymorphic loci were G, G, A, and $\mathrm{T}$ and the frequency of the heterozygous genotype was higher than that of the homozygous genotype in Tianfu meat geese. Compared to that in the population of non-knob geese of Tianfu meat geese, the head dimensions in the population of knob geese were significantly higher except for nostril height. However, in the non-knob geese, beak width 1, beak width 2 , nostril length, cranial width 1 , and maxillary length had significant differences among different genotypes or haplotypes/diplotypes.

Conclusion: These results suggested that polymorphisms of the $\mathrm{DIO} 2$ gene could be considered molecular markers to select larger heads of geese in the population of non-knob geese.

Keywords: Deiodinase 2; Geese; Head Dimensions; Knob; Polymorphic Loci

\section{INTRODUCTION}

Deiodinase 2 (DIO2), predominantly converts prohormone T4 to active hormone T3, which plays important roles in several biological processes, such as the development of skeletal muscle, regulation of the hypothalamus-pituitary-thyroid axis, adaptive thermogenesis and metabolic control, behavior, and mood [1]. Evidence has shown that knockdown of the DIO2 gene could result in increased fat storage in adipose tissue, hepatic steatosis [2], and increased subchondral bone mineral content [3]. Bone derived from DIO2-knockout mice showed reduced toughness, was brittle and had increased vulnerability to fracture, emphasizing that $\mathrm{DIO} 2$ was an important prerequisite for optimal bone remodeling [4]. Notably, common variation in the DIO2 gene, particularly the Thr92Ala substitution, was associated with osteoarthritis and intelligence quotient [5-7]. The subjects carrying the DIO2-Thr92Ala polymorphism had consistently lower femoral neck and total hip densities than wild-type subjects, accompanied by significantly higher levels of bone turner markers, 
which indicated a role for the $\mathrm{DIO} 2$ gene in the regulation of bone formation [8]. Recent research has also shown that single nucleotide polymorphisms (SNPs) of DIO2 influence thyroid metabolism, resulting in neurological disease [9]. Thyroid hormones (THs) have pivotal roles in the development and growth of the craniofacial skeleton, and their action in target tissues is dependent on the intracellular concentration of T3, which is locally regulated by DIO2 and deiodinase 3 (DIO3) [10]. To date, the regulatory mechanisms of craniofacial malformation caused by $\mathrm{TH}$ deficiency or excess have mainly focused on mutations of a single gene [11,12]. As a key gene regulating the synthesis of THs, it was not clear whether the DIO2 gene affected the development and growth of the craniofacial skeleton.

As an economically important poultry, domestic geese have distinct appearance characteristics, especially in the head. Recent studies showed that the DIO2 gene was considered the crucial gene associated with geese's knob phenotype, a feature located at the base of the upper bill in Chinese native geese breeds and absent in European geese breeds, and the growth and development of the knob was mediated by the TH synthesis signaling pathway in geese $[13,14]$. Notably, the knob has impressive value, as a large knob phenotypic size is generally preferred in Chinese markets according to market research [13]. Therefore, the aim of the present study was to identify and compare the molecular characteristics of the DIO2 gene between Sichuan White geese (with a knob) and Landes geese (devoid of a knob) and then to analyze the association between polymorphisms of the $\mathrm{DIO} 2$ gene and the craniofacial dimensions in the Tianfu meat geese breed, which was constructed by crossing Sichuan White geese and Landes geese. This study was approved by the 6th (2015) China Waterfowl Development Conference. These results contribute to basic research on the craniofacial characteristics of geese and further breeding for the Tianfu meat geese breed.

\section{MATERIALS AND METHODS}

\section{Ethics approval}

This study was conducted according to the guidelines of the Institutional Animal Care and Use Committee (IACUC) of Sichuan Agricultural University (Chengdu campus, Sichuan, China, Permit No. DKY20170913).

\section{Animals and sample preparation}

Sichuan White geese, Landes geese, and Tianfu meat geese used in the present study were provided by the Waterfowl Breeding Experimental Farm of Sichuan Agricultural University (Yaan, Sichuan). The population of Tianfu meat geese has been artificially selected for over 10 generations and the craniofacial characteristics are significantly different. The geese were raised in floor pens with free access to water and food. Hypothalamus tissues from Sichuan White geese (3 years old, $\mathrm{n}=4$ ) and Landes geese ( 3 years old, $\mathrm{n}=4$ ) were used to clone the coding sequence of the $\mathrm{DIO} 2$ gene. Blood samples ( $5 \mathrm{~mL} /$ goose) from Tianfu meat geese were collected from the wing veins of all geese ( 3 years old, $n=350,75$ males; 275 females) into ethylenediaminetetraacetic acidcoated tubes for deoxyribonucleic acid (DNA) extraction.

\section{Ribonucleic acid extraction and molecular cloning}

Total ribonucleic acid (RNA) was isolated from the hypothalamus of Sichuan White geese and Landes geese using TRIzol reagent (Takara, Dalian, China). The purity and quality of RNA were detected by spectrophotometric absorbance at $260 / 280 \mathrm{~nm}$ and $260 / 230 \mathrm{~nm}$, respectively. The integrity of RNA was verified on a $1.5 \%$ agarose gel. Complementary DNA (cDNA) was synthesized from $1 \mu \mathrm{g}$ of total RNA using a cDNA synthesis kit (Takara, China) according to the manufacturer's instructions. The polymerase chain reaction (PCR) was performed in a total volume of $10 \mu \mathrm{L}$ using $5 \mu \mathrm{L}$ of $2 \times$ PCR HeroTM Mix(dye) (FOREGENE, Chengdu, China), 1 $\mu \mathrm{L}$ of cDNA, $0.2 \mu \mathrm{L}$ of primers $(10 \mu \mathrm{M}$ each $)$ and $3.6 \mu \mathrm{L}$ of $\mathrm{ddH}_{2} \mathrm{O}$. PCR was performed as follows: $94^{\circ} \mathrm{C}$ predenaturation for $3 \mathrm{~min}, 35$ cycles at $94^{\circ} \mathrm{C}$ denaturation for $10 \mathrm{~s}$, annealing temperature for $10 \mathrm{~s}$, and $72^{\circ} \mathrm{C}$ extension for $20 \mathrm{~s}$, with a final extension at $72^{\circ} \mathrm{C}$ for $5 \mathrm{~min}$. The PCR products were gelpurified using a gel extraction kit (OMEGA, Norwalk, CT, USA). Target cDNA was ligated into the $\mathrm{pMD}-19 \mathrm{~T}$ vector (Takara, China) and was then transformed into Escherichia coli DH5a competent cells. Positive clones that contained the expected-size inserts were screened by using colony PCR and were then sequenced by Qinke Gene Biotechnology Co. Ltd. (Chengdu, China). Primers of the DIO2 gene are shown in Supplementary Table S1.

\section{Bioinformatical analysis}

VecScreen (https://www.ncbi.nlm.nih.gov/tools/vecscreen/) was used to search for vector contamination. The BLASTn program in the National Center for Biotechnology Information (NCBI) was used to analyze the accuracy of cloning sequences (https://blast.ncbi.nlm.nih.gov/Blast.cgi). DNAMAN software was used to analyze the homologies of nucleotide sequences and to identify the polymorphic loci of the DIO2 gene between Sichuan White geese and Landes geese by sequence alignment. MEGA 7.0 software was used to construct a phylogenetic tree by the neighbor-joining method with 1,000 bootstrap replicates.

\section{Polymorphic loci validation and genotype in Tianfu meat geese}

Tianfu meat geese needed to continue to be used in production; therefore, genomic DNA of each individual was extracted 
from whole blood using an Animal Tissue DNA Isolation Kit (DE-05012, FOREGENE, China) following the manufacturer's protocol. Three pairs of primers were designed for screening the polymorphic loci located at the coding sequence of the DIO2 gene in Tianfu meat geese (Supplementary Table S1). The reaction system was performed in a total volume of 20 $\mu \mathrm{L}$ using $10 \mu \mathrm{L}$ of $2 \times$ PCR HeroTM Mix(dye) (FOREGENE, China), $2 \mu \mathrm{L}$ of DNA, $0.4 \mu \mathrm{L}$ of primers $(10 \mu \mathrm{M}$ each) and $7.2 \mu \mathrm{L}$ of $\mathrm{ddH}_{2} \mathrm{O}$. The PCR conditions were $94^{\circ} \mathrm{C}$ predenaturation for $3 \mathrm{~min}, 35 \mathrm{cycles}$ at $94^{\circ} \mathrm{C}$ denaturation for $10 \mathrm{~s}$, annealing temperature for $10 \mathrm{~s}, 72^{\circ} \mathrm{C}$ extension $20 \mathrm{~s}$, and a final extension at $72^{\circ} \mathrm{C}$ for $5 \mathrm{~min}$. The PCR products were examined by $1.5 \%$ agarose gel electrophoresis and then sequenced by Qinke Gene Biotechnology Co. Ltd. (China).

\section{The measurement of head dimensions in Tianfu meat geese}

The body weight of Tianfu meat geese was recorded, and the head dimensions were measured individually. The measurement standards of head dimensions are shown in Figure 1A and $1 \mathrm{~B}$. These indices included cranial length $(\mathrm{CL}$, length from occipital bone to the junction between the frontal bone and the nasal bone), maxillary length (ML, length from the end of the quadratojugal bone to the tip of the beak), beak length 2 (BL2, length from the tip of the quadratojugal bone to the tip of the beak), nostril length (NL, length between the upper and lower nostril), nostril height $(\mathrm{NH}$, height of the center of the nasal bone perpendicular to the nostril), height of upper beak 1 (UBH1, height of the junction between the frontal bone and the nasal bone to the tip of the quadratojugal bone), height of upper beak 2 (UBH2, height of the center of the nasal bone perpendicular to the upper beak), beak length 1 (BL1, length from the junction between the frontal bone and the nasal bone to the tip of the beak), cranial width 1 (CW1, breadth of the junction across the orbits and the frontal bone), cranial width 2 (CW2, smallest breadth between the orbits on the dorsal side), cranial width 3 (CW3, breadth across the protuberentia occipitalis externa), beak width 1 (BW1, breadth of the junction between the frontal bone and the nasal bone), beak width 2 (BW2, breadth of the center of the nasal bone), and beak width 3 (BW3, breadth across the beak bean). Centimeter $(\mathrm{cm})$ was used as the unit for length, height, and width.

\section{Statistical analysis}

In the present study, Tianfu meat geese individuals whose body weights were more than twice the standard deviations from the mean were removed, and several individuals with uncertain knobs were also eliminated. Ultimately, 304 individuals (60 males; 244 females) were used for further analysis. The sequencing results were viewed in BioEdit software (https:// bitesizebio.com/10238/bioedit-a-sequence-alignment-editorand-it-is-free/) to ensure the polymorphic loci of the DIO2 gene. The information of allele and genotype of $\mathrm{DIO} 2$ gene was sorted in Excel 2019. Allelic and genotypic frequencies were determined by direct counting. Population indices, including heterozygosity $(\mathrm{He})$, homozygosity $(\mathrm{Ho})$, and effective number of alleles $(\mathrm{Ne})$ were calculated by the following formulas:

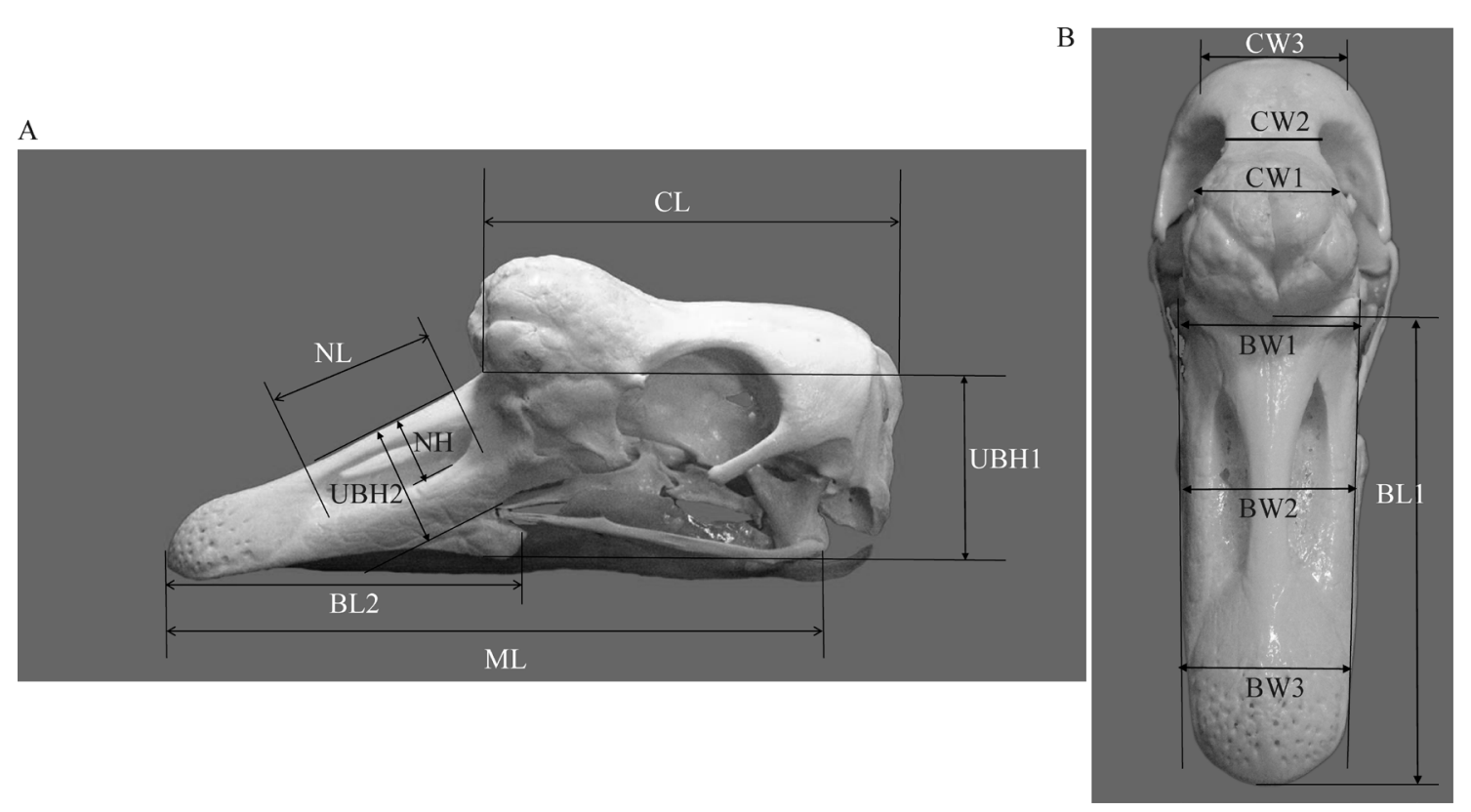

Figure 1. Length, height and width measurement of the goose head. (A) Lateral aspect image. $C L$, cranial length; $M L$, maxillary length; $B L 2$, beak length 2; NL, nostril length; UBH1, height of upper beak 1; UBH2, height of upper beak 2; NH, nostril height. (B) Dorso-ventral aspect image. BL1, beak length 1; CW1, cranial width 1; CW2, cranial width 2; CW3, cranial width 3; BW1, beak width 1; BW2, beak width 2; BW3, beak width 3 . 


$$
H o=\sum_{i=1}^{n} p_{i}^{2} ; H e=1-H o ; N e=1 / H o
$$

$P_{i}$ represents the i-th allelic frequency at a locus, and $n$ represents the number of alleles at a locus.

The polymorphism information content (PIC) was directly calculated by PIC_CALC 0.6 software. The polymorphic loci of the DIO2 gene were subjected to linkage disequilibrium (LD) analysis using the SHEsis platform and to haplotype/ diplotype analysis by Haploview 4.1. The haplotypes and dipoltypes with frequencies below $5 \%$ were not subjected to subsequent analysis.

The general linear model (GLM) procedure of SPSS. 24.0 was used to test the association between the knob phenotype and head dimensions. The model was as follows:

$$
Y_{i j}=\mu+\mathrm{G}_{i}+\mathrm{S}_{j}+\mathrm{e}_{i j}
$$

where $Y_{i j}$ is the measurement of a trait, $\mu$ is the overall population mean, $G_{i}$ is the fixed effect of phenotype $(i=2), S_{j}$ is the fixed effect of $\operatorname{sex}(j=2)$ and $e_{i j}$ is the random error. The values are presented as the mean \pm standard deviation. Multiple comparisons were carried out using analysis of variance followed by Duncan's test using SPSS. 24.0. An independent nonpaired t-test analysis (two-tailed) was used for comparisons between the two groups. Statistical significance was considered at $\mathrm{p}<0.05$.

\section{RESULTS}

$\mathrm{DIO} 2$ gene cloning and sequence comparison analysis In the present study, the coding sequences of the DIO2 gene of both Sichuan White geese and Landes geese were cloned. The sequence of the DIO2 gene consisted of 840 nucleotides (Figure 2). Homology analysis of nucleotide sequences showed that there was high sequence homology (95.42\%) between geese and other avian species, such as chicken and quail, intermediate homology (88.06\%) between geese and fish, such as zebrafish, and relatively less homology (86.95\%) between geese and mammals, such as humans and mice. In addition, a phylogenetic tree was constructed based on the DIO2 nucleotide acid sequences for geese and other species (Supplementary Figure S1), which indicated that the DIO2 genes in Sichuan White geese and Landes geese were similar to the DIO2 genes of other avians. Furthermore, the sequences of the DIO2 genes of Sichuan White geese and Landes geese were aligned, and four polymorphic loci, g.419 A>G, g.533 A>G, g.725 C>A, and g.799 C>T, were identified. Among these loci, the g.799 C $>$ T locus changed the amino acid from lysine to proline.

Genetic parameter analysis of $\mathrm{DIO} 2$ gene polymorphisms

\section{in Tianfu meat geese}

To identify the genetic parameters of DIO2 gene polymorphisms, a crossbred population of Tianfu meat geese was used. Genotype analysis showed that the g.419 A>G, g.533 A>G, g.725 C>A, and g.799 C>T loci had three genotypes, AA/ GG/GA, AA/GG/GA, CC/AA/AC, and CC/TT/TC, in Tianfu meat geese (Figure 3). The genotypic and allelic frequencies as well as estimated population indices (heterozygosity, effective allele numbers, PIC) were calculated and were shown in Supplementary Table S2. The results showed that the dominant alleles at the g.419A $>$ G, g.533A $>$ G, g.725C $>A$, and g.799C $>T$ loci were $G, G, A$, and $T$, respectively. The frequencies of heterozygous genotypes of four polymorphic loci in this population were higher than those of homozygous genotypes. The estimation of population indices showed that the values of $\mathrm{Ho}$ and $\mathrm{He}$ among g.419 A>G, g.533 A>G, g.799 C>T loci were consistent, in which $\mathrm{Ho}$ was observed as 0.50 , He was observed as 0.50 , and $\mathrm{Ne}$ was observed as 1.98 , while at the g.725 C>A locus, Ho was 0.51, He was 0.49, and Ne was 1.97 . According to the classification of PIC, the four polymorphic loci of the DIO2 gene in this population possessed intermediate polymorphisms $(0<\mathrm{PIC}<0.5)$. The chi-square test showed that the genotypic frequencies of the four polymorphic loci were in accordance with Hardy-Weinberg equilibrium ( $\mathrm{p}>$ 0.05).

\section{Association analysis between knob phenotype and the} head dimensions of geese

To investigate whether the knob and non-knob phenotypes influenced the head dimensions of geese, a comparison analysis of head dimensions was carried out between knob and non-knob goose populations. The results showed that there was no interaction between the fixed effect of sex and the knob and non-knob phenotypes. Compared to that in the population of non-knob geese, the head dimensions in the population of knob geese were significantly higher except for NH (Table 1, p<0.05).

Association analysis between polymorphisms of the $\mathrm{DIO} 2$ gene and the head dimensions of geese

As stated in the previous results, the $\mathrm{DIO} 2$ gene was crucial to regulate the knob and non-knob phenotypes, and the knob phenotype was related to the head dimensions of geese. Thus, the population of Tianfu meat geese was divided into populations of knob and non-knob geese, and the association analysis of polymorphisms of the DIO2 gene with the head dimensions of geese was carried out in populations of knob and non-knob geese. In the population of knob geese, as shown in Table 2, the different genotypes of polymorphisms of the DIO2 gene were not significantly different from the head dimensions of geese $(\mathrm{p}>0.05)$. Further analysis found that individuals with the AA genotype at the g.419A $>\mathrm{G}$ and 
ATGGGTCT GCTAAGT GTGGATTTGCTGATCACGCTTCAGATCTTGCCGGTCTTTTTCTCC

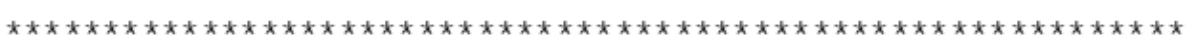
ATGGGTCTGCTAAGTGTGGATTTGCTGATCACGCTTCAGATCTTGCCGGTCTTTTTCTCC AATTGCCTCTTCCTTGCACTCTATGACTCTGTGATCCTCCTGAAGCACATGGTGCTGTTC

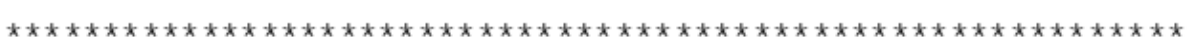

CTGAGCCGCTCCAAGGCTGCGCGCGGCGAGTGGCGGAGGATGCT CACCTCGGAGGGGCTG

181 CGCTGCGTCTGGAACAGCTTCCTCCTGGACGCCTACAAGCAGGTCAAATTGGGAGGAGAA

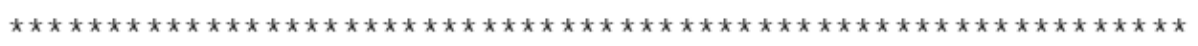

181 CGCTGCGTCTGGAACAGCTTCCTCCTGGACGCCTACAAGCAGGTCAAATTGGGAGGAGAA

241 GCCCCAAACTCCAGTGTAATCCACATAGCCAAGGGCAGCGATGGCAGCAGTAGCAGCTGG $* * * * * * * * * * * * * * * * * * * * * * * * * * * * * * * * * * * * * * * * * * * * * * * * * * * * * * * * * * * * * *$

241 GCCCCAAACTCCAGTGTAATCCACATAGCCAAGGGCAGCGATGGCAGCAGTAGCAGCTGG 301 AAGAGTGTTGGTGGCAAGTGTGGAACCAAAT GCCACCTTCTGGATTTCGCCAACTCTGAG

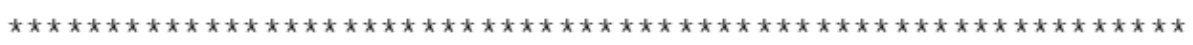

301 AAGAGTGTTGGTGGCAAGTGTGGAACCAAAT GCCACCTTCTGGATTTCGCCAACTCTGAG 361 CGGCCACTGGTGGTCAACTTTGGTTCAGCTACCTGACCTCCATTCACAAGCCAGCTGTCA $* * * * * * * * * * * * * * * * * * * * * * * * * * * * * * * * * * * * * * * * * * * * * * * * * * * * * * * * * * * * *$

361 CGGCCACTGGTGGTCAACTTTGGTTCAGCTACCTGACCTCCATTCACAAGCCAGCTGTCG 421 GCCTTCAGCAAGCTGGTGGAAGAGTTCTCTGTGTGGCTGACTTTCTGTTGGTCTACATT

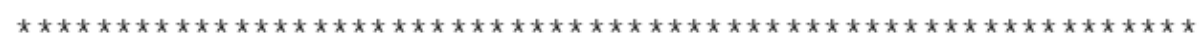

421 GCCTTCAGCAAGCTGGTGGAAGAGTTCTCTGGTGTGGCTGACTTTCTGTTGGTCTACATT

481 GATGAGGCTCACCCGTCAGATGGCTGGGCTGCCCCTGGAATCTCTCCCTCTTCATTTGAA **********************************************************************

481 GATGAGGCTCACCCGTCAGATGGCTGGGCTGCCCCTGGAATCTCTCCCTCTTCGTTTGAA 541 GTTAAGAAACACAGAAACCAGGAAGATCGATGCGCAGCTGCTCACCAACTCCTAGAGCGC

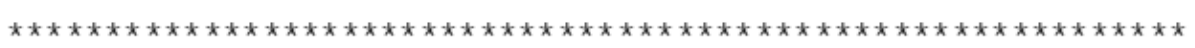

541 GTTAAGAAACACA GAAACCAGGAAGATCGAT GCGCAGCTGCTCACCAACTCCTAGAGCGC 601 TTCTCCTTGCCACCTCAGTGCCAGGTGGTGGCTGACTGCATGGACAACAATGCCAATGTG $* * * * * * * * * * * * * * * * * * * * * * * * * * * * * * * * * * * * * * * * * * * * * * * * * * * * * * * * * * * *$

601 TTCTCCTTGCCACCTCAGTGCCAGGTGGTGGCTGACTGCATGGACAACAATGCCAATGTG 661 GCCTATGGGGTTTCATTTGAACGAGTATGCATTGTGCAGAGACAAAAAATCGCCTATCTA

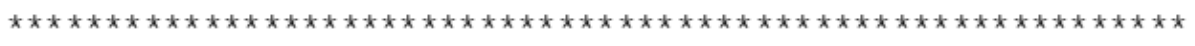

661 GCCTATGGGGTTCATTTGAACGAGTATGCATTGTGCAGAGACAAAAAATCGCCTATCTA 721 GGAGGCAAAGGCCCCTTTTTCTACAATCTGCAGGAGGTTCGGCTTTGGCTGGAACAAAAT

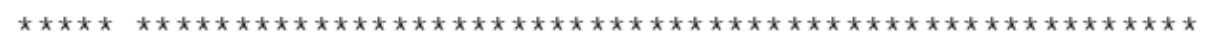
721 GGAGGAAAAGGCCCCTTTTTCTACAATCTGCAGGAGGTTCGGCTTTGGCTGGAACAAAAT 781 TTCAGCAAAAGATGAAATCCATTTCCGACAGAAGATATGTCAACAGGTGTGTCCTTTTAA

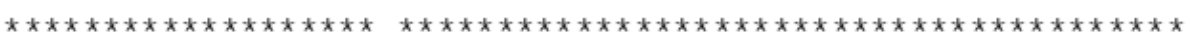

781 TTCAGCAAAAGATGAAATCTATTTCCGACAGAAGATATGTCAACAGGTGTGTCCTTTTAA

Figure 2. Comparison analysis of the deiodinase 2 gene coding sequences between Sichuan White geese and Landes geese. The top row represented Landes geese, the bottom row represented Sichuan White geese. * Indicated the consistent nucleotides, locations at 419, 533, 725, 799 indicated the inconsistent nucleotides. The number on the left represented the position of nucleotides. 

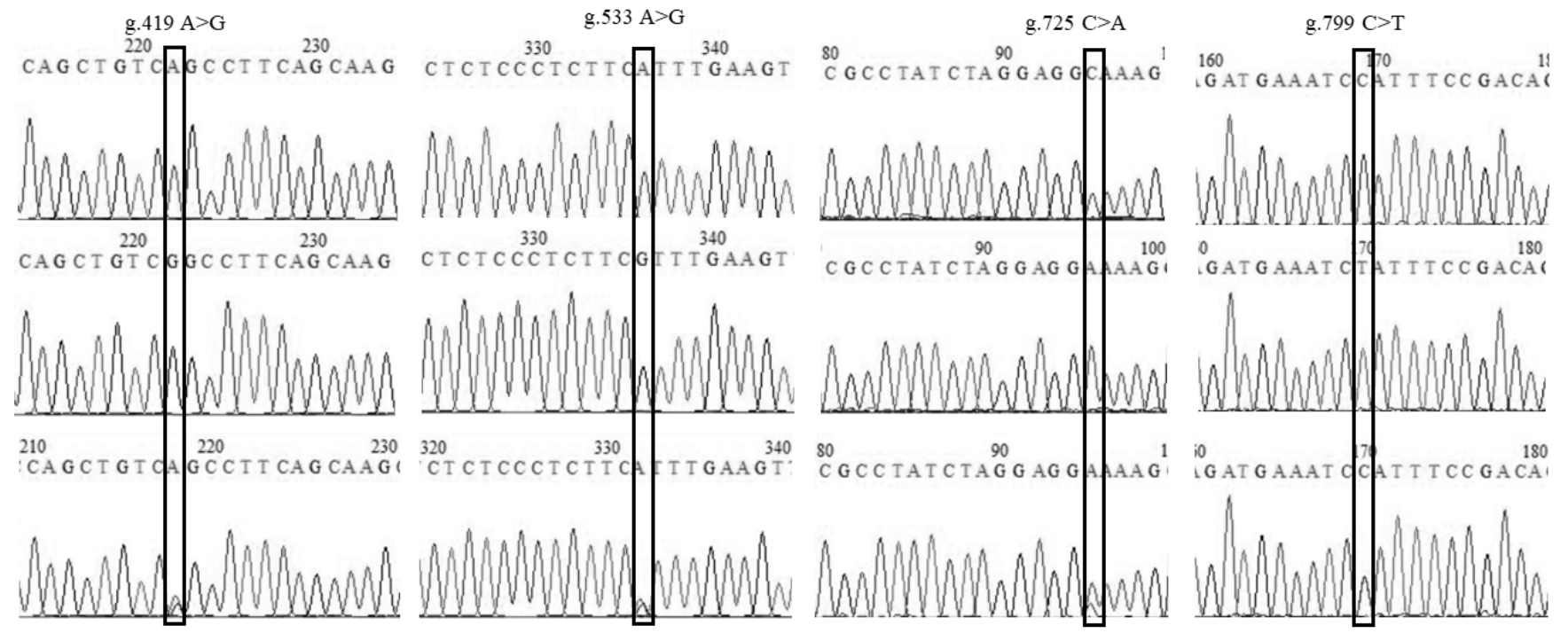

Figure 3. Confirmation of the four polymorphic loci of the deiodinase 2 gene in the population of Tianfu meat geese. The black boxes represented the polymorphic loci. The first and second rows were homozygotes, and the third row was heterozygote.

Table 1. Association analysis between the knob and the head dimensions in goose

\begin{tabular}{|c|c|c|c|c|c|c|c|c|c|c|c|c|c|c|}
\hline Knob & BL1 & BL2 & BW1 & BW2 & BW3 & CW1 & CW2 & CW3 & ML & $\mathrm{CL}$ & UBH1 & $\mathrm{UBH} 2$ & NL & NH \\
\hline & & $7.19 \pm 0.57$ & & $2.74 \pm 0.17^{\star}$ & & $2.89 \pm 0.26^{\star}$ & $2.29 \pm 0.22^{\star}$ & $3.08 \pm 0.24^{\star}$ & & $8.16 \pm 0.45^{\star}$ & & & & \\
\hline Non-knob geese (166) & $6.29 \pm 0.39$ & $6.89 \pm 0.46$ & $3.01 \pm 0.18$ & $2.61 \pm 0.14$ & $2.41 \pm 0.10$ & $2.73 \pm 0.18$ & $2.16 \pm 0.19$ & $2.94 \pm 0.25$ & $13.25 \pm 0.66$ & $7.91 \pm 0.36$ & $3.01 \pm 0.24$ & $1.78 \pm 0.19$ & $2.83 \pm 0.22$ & $0.80 \pm 0.14$ \\
\hline
\end{tabular}

The number behind the knob types represented the number of individuals. Centimeter $(\mathrm{cm})$ was used as the unit for length, height and width

BL1, beak length 1; BL2, beak length 2; BW1, beak width 1; BW2, beak width 2; BW3, beak width 3; CW1, cranial width 1; CW2, cranial width 2; CW3, cranial width 3; ML, maxillary length; CL, cranial length; $\mathrm{UBH} 1$, height of upper beak 1 ; UBH2, height of upper beak 2; NL, nostril length; NH, nostril height.

* Represented the statistical significance within the same column differ significantly at $p<0.05$

Table 2. Association analysis between polymorphic loci of the deiodinase 2 gene and the head dimensions in knob geese

\begin{tabular}{|c|c|c|c|c|c|c|c|c|c|c|c|c|c|c|c|}
\hline Loci & Genotypes & BL1 & BL2 & BW1 & BW2 & BW3 & CW1 & CW2 & CW3 & ML & CL & UBH1 & UBH2 & NL & NH \\
\hline \multirow[t]{3}{*}{ g. $419 A>G$} & GG (40) & $6.65 \pm 0.46$ & $7.23 \pm 0.50$ & $3.17 \pm 0.20$ & $2.74 \pm 0.18$ & $2.51 \pm 0.16$ & $2.85 \pm 0.21$ & $2.25 \pm 0.20$ & $3.07 \pm 0.22$ & $13.77 \pm 0.85$ & $8.19 \pm 0.39$ & $3.17 \pm 0.27$ & $1.88 \pm 0.20$ & $2.92 \pm 0.21$ & $0.80 \pm 0.16$ \\
\hline & AA (27) & $6.67 \pm 0.52$ & $7.19 \pm 0.60$ & $3.20 \pm 0.20$ & $2.71 \pm 0.15$ & $2.51 \pm 0.14$ & $2.93 \pm 0.27$ & $2.24 \pm 0.25$ & $3.06 \pm 0.27$ & $13.89 \pm 0.88$ & $8.11 \pm 0.48$ & $3.15 \pm 0.29$ & $1.90 \pm 0.27$ & $2.92 \pm 0.22$ & $0.82 \pm 0.19$ \\
\hline & GA (71) & $6.59 \pm 0.54$ & $7.17 \pm 0.61$ & $3.18 \pm 0.21$ & $2.75 \pm 0.18$ & $2.52 \pm 0.15$ & $2.90 \pm 0.29$ & $2.33 \pm 0.22$ & $3.09 \pm 0.25$ & $13.78 \pm 0.96$ & $8.16 \pm 0.48$ & $3.10 \pm 0.27$ & $1.87 \pm 0.27$ & $2.90 \pm 0.26$ & $0.82 \pm 0.15$ \\
\hline \multirow[t]{3}{*}{ g. $533 A>G$} & GG (40) & $6.65 \pm 0.46$ & $7.23 \pm 0.50$ & $3.17 \pm 0.20$ & $2.74 \pm 0.18$ & $2.51 \pm 0.16$ & $2.85 \pm 0.21$ & $2.25 \pm 0.20$ & $3.07 \pm 0.22$ & $13.77 \pm 0.85$ & $8.19 \pm 0.39$ & $3.17 \pm 0.27$ & $1.88 \pm 0.20$ & $2.92 \pm 0.21$ & $0.80 \pm 0.16$ \\
\hline & $\mathrm{AA}(27)$ & $6.67 \pm 0.52$ & $7.19 \pm 0.60$ & $3.20 \pm 0.20$ & $2.71 \pm 0.15$ & $2.51 \pm 0.14$ & $2.93 \pm 0.27$ & $2.24 \pm 0.25$ & $3.06 \pm 0.27$ & $13.89 \pm 0.88$ & $8.11 \pm 0.48$ & $3.15 \pm 0.29$ & $1.90 \pm 0.27$ & $2.92 \pm 0.22$ & $0.82 \pm 0.19$ \\
\hline & GA (71) & $6.59 \pm 0.54$ & $7.17 \pm 0.61$ & $3.18 \pm 0.21$ & $2.75 \pm 0.18$ & $2.52 \pm 0.15$ & $2.90 \pm 0.29$ & $2.33 \pm 0.22$ & $3.09 \pm 0.25$ & $13.78 \pm 0.96$ & $8.16 \pm 0.48$ & $3.10 \pm 0.27$ & $1.87 \pm 0.27$ & $2.90 \pm 0.26$ & $0.82 \pm 0.15$ \\
\hline \multirow[t]{3}{*}{ g. $725 C>A$} & AA (43) & $6.59 \pm 0.52$ & $7.20 \pm 0.51$ & $3.15 \pm 0.21$ & $2.73 \pm 0.18$ & $2.49 \pm 0.16$ & $2.85 \pm 0.20$ & $2.26 \pm 0.19$ & $3.07 \pm 0.23$ & $13.70 \pm 0.89$ & $8.15 \pm 0.42$ & $3.12 \pm 0.31$ & $1.85 \pm 0.24$ & $2.88 \pm 0.24$ & $0.80 \pm 0.16$ \\
\hline & CC (25) & $6.67 \pm 0.50$ & $7.25 \pm 0.55$ & $3.21 \pm 0.21$ & $2.72 \pm 0.15$ & $2.51 \pm 0.14$ & $2.95 \pm 0.27$ & $2.25 \pm 0.26$ & $3.09 \pm 0.27$ & $13.89 \pm 0.90$ & $8.13 \pm 0.47$ & $3.16 \pm 0.30$ & $1.91 \pm 0.28$ & $2.93 \pm 0.23$ & $0.82 \pm 0.19$ \\
\hline & AC (70) & $6.64 \pm 0.51$ & $7.19 \pm 0.60$ & $3.19 \pm 0.21$ & $2.76 \pm 0.18$ & $2.53 \pm 0.14$ & $2.90 \pm 0.29$ & $2.32 \pm 0.22$ & $3.08 \pm 0.25$ & $13.83 \pm 0.93$ & $8.17 \pm 0.47$ & $3.12 \pm 0.25$ & $1.88 \pm 0.25$ & $2.92 \pm 0.25$ & $0.82 \pm 0.14$ \\
\hline \multirow[t]{3}{*}{ g.799 C> $>$} & TT (41) & $6.62 \pm 0.51$ & $7.23 \pm 0.50$ & $3.16 \pm 0.21$ & $2.75 \pm 0.18$ & $2.51 \pm 0.17$ & $2.85 \pm 0.20$ & $2.26 \pm 0.19$ & $3.08 \pm 0.22$ & $13.73 \pm 0.88$ & $8.20 \pm 0.41$ & $3.16 \pm 0.28$ & $1.89 \pm 0.20$ & $2.92 \pm 0.21$ & $0.80 \pm 0.16$ \\
\hline & CC (26) & $6.64 \pm 0.51$ & $7.19 \pm 0.62$ & $3.20 \pm 0.21$ & $2.72 \pm 0.15$ & $2.51 \pm 0.14$ & $2.93 \pm 0.27$ & $2.24 \pm 0.25$ & $3.08 \pm 0.27$ & $13.87 \pm 0.89$ & $8.14 \pm 0.47$ & $3.15 \pm 0.30$ & $1.90 \pm 0.27$ & $2.92 \pm 0.23$ & $0.82 \pm 0.19$ \\
\hline & TC (71) & $6.62 \pm 0.52$ & $7.17 \pm 0.61$ & $3.19 \pm 0.21$ & $2.75 \pm 0.18$ & $2.52 \pm 0.14$ & $2.90 \pm 0.29$ & $2.32 \pm 0.22$ & $3.08 \pm 0.25$ & $13.81 \pm 0.94$ & $8.14 \pm 0.48$ & $3.10 \pm 0.26$ & $1.85 \pm 0.27$ & $2.90 \pm 0.26$ & $0.82 \pm 0.14$ \\
\hline
\end{tabular}

The number behind the genotype represented the number of individuals. Centimeter (cm) was used as the unit for length, height and width.

BL1, beak length 1; BL2, beak length 2; BW1, beak width 1; BW2, beak width 2; BW3, beak width 3; CW1, cranial width 1; CW2, cranial width 2; CW3, cranial width 3; ML, maxillary length; CL, cranial length;

$\mathrm{UBH} 1$, height of upper beak 1 ; UBH2, height of upper beak 2; NL, nostril length; $\mathrm{NH}$, nostril height.

g.533A $>\mathrm{G}$ loci or the CC genotype at the g.725C >A and g.799C $>$ T loci had higher BL1, BW1, CW1, ML, UBH2, and $\mathrm{NH}$ than those with the GG genotype at the g.419A $>\mathrm{G}$ and g.533A $>\mathrm{G}$ loci, AA genotype at the g.725C $>\mathrm{A}$ locus and TT genotype at the g.799C $>$ T locus $(\mathrm{p}>0.05)$. However, in the population of non-knob geese, as shown in Table 3, individuals with the AA genotype at the g.419A $>\mathrm{G}$ and g.533A $>\mathrm{G}$ loci or the CC genotype at the g.725C $>\mathrm{A}$ and g.799C $>$ T loci were significantly higher in BW1, BW2, and $\mathrm{NL}$ than in other genotypes $(\mathrm{p}<0.05)$. Individuals with the CC genotype at the g.725C $>\mathrm{A}$ and g.799C $>\mathrm{T}$ loci were sig- nificantly wider in CW1 than in other genotypes $(p<0.05)$. At the g.419A $>\mathrm{G}$ and g.533A $>\mathrm{G}$ loci, individuals with the AA genotype were significantly longer in ML than those with other genotypes, while individuals with the GA genotype were significantly higher in $\mathrm{NH}$ than those with other genotypes $(\mathrm{p}<0.05)$.

\section{Association analysis between haplotypes/diplotypes and the head dimensions of geese}

The LD test showed that the four polymorphic loci of the DIO2 gene were strongly linked in the population of non- 
Table 3. Association analysis between polymorphic loci of the deiodinase 2 gene and the head dimensions in non-knob geese

\begin{tabular}{|c|c|c|c|c|c|c|c|c|c|c|c|c|c|c|c|}
\hline Loci & eno & L1 & BL2 & W1 & W2 & 3W3 & W1 & W2 & CW3 & ML & CL & UBH1 & UBH2 & NL & NH \\
\hline \multirow[t]{3}{*}{ g. $419 \mathrm{G}>\mathrm{A}$} & GG (56) & $32 \pm 0.39$ & $2 \pm 0.47$ & & .15 & & $0 \pm 0.21$ & & 0.23 & 67 & $91 \pm 0.39$ & $2 \pm 0.24$ & & $2.82 \pm 0.21$ & $80 \pm 0.13$ \\
\hline & $\mathrm{AA}(40)$ & $6.33 \pm 0.38$ & $92 \pm 0.39$ & $3.09 \pm 0.19 *$ & $2.66 \pm 0.15^{\star}$ & $2.43 \pm 0.12$ & $2.79 \pm 0.16$ & $2.19 \pm 0.19$ & $2.91 \pm 0.31$ & $13.39 \pm 0.69 *$ & $7.99 \pm 0.32$ & $3.03 \pm 0.26$ & $1.75 \pm 0.17$ & $2.92 \pm 0.19 *$ & $0.76 \pm 0.10$ \\
\hline & & $6.25 \pm 0.39$ & $86 \pm 0.49$ & $2.98 \pm 0.16$ & $2.59 \pm 0.13$ & $2.40 \pm 0.10$ & $2.72 \pm 0.17$ & $2.16 \pm 0.20$ & $2.95 \pm 0.23$ & $13.13 \pm 0.61$ & $7.86 \pm 0.35$ & $2.98 \pm 0.24$ & $1.80 \pm 0.21$ & $2.79 \pm 0.23$ & $182 \pm 0.16^{\star}$ \\
\hline \multirow[t]{3}{*}{ g. $533 \mathrm{G}>\mathrm{A}$} & GG (56) & & $92 \pm C$ & $3.00 \pm 0.18$ & $2.60 \pm 0.15$ & $2.40 \pm 0.10$ & $2.70 \pm 0.21$ & & $2.96 \pm 0.23$ & $13.31 \pm 0.67$ & $7.91 \pm 0.39$ & & & & $80 \pm 0.13$ \\
\hline & $\mathrm{AA}(40)$ & $6.33 \pm 0.38$ & $6.92 \pm 0.39$ & $3.09 \pm 0.19 *$ & $2.66 \pm 0.15^{\star}$ & $2.43 \pm 0.12$ & $2.79 \pm 0.16$ & $2.19 \pm 0.19$ & $2.91 \pm 0.31$ & $13.39 \pm 0.69 *$ & $7.99 \pm 0.32$ & $3.03 \pm 0.26$ & $1.75 \pm 0.17$ & $2.92 \pm 0.19^{\star}$ & $0.76 \pm 0.10$ \\
\hline & GA (70) & $6.25 \pm 0.39$ & $6.86 \pm 0.49$ & $2.98 \pm 0.16$ & $2.59 \pm 0.13$ & $2.40 \pm 0.10$ & $2.72 \pm 0.17$ & $2.16 \pm 0.20$ & $2.95 \pm 0.23$ & $13.13 \pm 0.61$ & $7.86 \pm 0.35$ & $2.98 \pm 0.24$ & $1.80 \pm 0.21$ & 23 & $0.16^{*}$ \\
\hline \multirow[t]{3}{*}{ g. $725 \mathrm{~A}>C$} & AA (56) & $6.32 \pm 0.39$ & $6.91 \pm 0.47$ & $2.99 \pm 0.18$ & $2.61 \pm 0.14$ & $2.41 \pm 0.11$ & $2.70 \pm 0.21$ & $2.16 \pm 0.19$ & $2.97 \pm 0.23$ & $13.25 \pm 0.63$ & $7.94 \pm 0.36$ & $3.02 \pm 0.25$ & $1.78 \pm$ & $2.81 \pm 0.21$ & $0.81 \pm 0.13$ \\
\hline & CC (39) & $6.32 \pm 0.38$ & $6.91 \pm 0.39$ & $3.09 \pm 0.19 *$ & $2.65 \pm 0.15^{\star}$ & $2.43 \pm 0.11$ & $2.78 \pm 0.16^{\star}$ & $2.19 \pm 0.18$ & $2.91 \pm 0.31$ & $13.36 \pm 0.68$ & $7.99 \pm 0.32$ & $3.04 \pm 0.26$ & $1.75 \pm 0.17$ & $2.92 \pm 0.19^{*}$ & $0.76 \pm 0.10$ \\
\hline & $A C(71)$ & $6.25 \pm 0.39$ & $6.87 \pm 0.48$ & $2.99 \pm 0.15$ & $2.59 \pm 0.13$ & $2.40 \pm 0.10$ & $2.72 \pm 0.17$ & $2.15 \pm 0.20$ & $2.94 \pm 0.23$ & $13.19 \pm 0.66$ & $7.85 \pm 0.37$ & $2.98 \pm$ & $1.79 \pm 0.21$ & $2.80 \pm 0.23$ & $0.81 \pm 0.16$ \\
\hline \multirow[t]{3}{*}{ g.799 T>C } & $\mathrm{TT}(55)$ & $6.33 \pm 0.38$ & $6.94 \pm 0.46$ & $3.01 \pm 0.18$ & $2.62 \pm 0.14$ & $2.41 \pm 0.11$ & $2.71 \pm 0.21$ & $2.16 \pm 0.19$ & $2.96 \pm 0.23$ & $13.32 \pm 0.66$ & $7.92 \pm 0.39$ & $3.02 \pm 0.24$ & $1.78 \pm 0.18$ & $2.82 \pm 0.21$ & $0.81 \pm 0.13$ \\
\hline & CC (39) & $6.32 \pm 0.38$ & $6.91 \pm 0.39$ & $3.09 \pm 0.19 *$ & $2.65 \pm 0.15^{\star}$ & $2.43 \pm 0.11$ & $2.78 \pm 0.16^{\star}$ & $2.19 \pm 0.18$ & $2.91 \pm 0.31$ & $13.36 \pm 0.68$ & $7.99 \pm 0.32$ & $3.04 \pm 0.26$ & $1.75 \pm 0.17$ & $2.92 \pm 0.19^{*}$ & $0.76 \pm 0.10$ \\
\hline & TC (72) & $6.24 \pm 0.40$ & $6.84 \pm 0.49$ & $2.98 \pm 0.16$ & $2.58 \pm 0.13$ & $2.39 \pm 0.10$ & $2.72 \pm 0.17$ & $2.15 \pm 0.20$ & $2.95 \pm 0.23$ & $13.13 \pm 0.63$ & $7.86 \pm 0.34$ & $2.98 \pm 0.24$ & $1.79 \pm 0.21$ & $2.79 \pm 0.23$ & $0.81 \pm 0.14$ \\
\hline
\end{tabular}

The number behind the genotype represented the number of individuals. Centimeter $(\mathrm{cm})$ was used as the unit for length, height and width

BL1, beak length 1; BL2, beak length 2; BW1, beak width 1; BW2, beak width 2; BW3, beak width 3; CW1, cranial width 1; CW2, cranial width 2; CW3, cranial width 3; ML, maxillary length; CL, cranial length; $\mathrm{UBH} 1$, height of upper beak 1; UBH2, height of upper beak 2; $\mathrm{NL}$, nostril length; $\mathrm{NH}$, nostril height

* Represented the statistical significance, $p<0.05$

knob geese (Supplementary Figure S2). The haplotype analysis showed that two different haplotypes were identified: GGAT with 53.3\% frequency and AACC with $43.7 \%$ frequency (Supplementary Table S3). Based on the haplotype analysis results, three major available diplotypes, GGAT-GGAT, GGAT-AACC, and AACC-AACC, were identified (Supplementary Table S3). Further association analysis showed that individuals with the AACC haplotype were significantly higher in BW1, CW1 and NL than in GGAT (Table 4, p<0.05), while individuals with the GGAT haplotype were significantly higher in $\mathrm{NH}$ than in AACC (Table 4, $\mathrm{p}<0.05$ ). Diplotype analysis showed that individuals with AACC-AACC diplotypes were significantly higher in BW1, BW2, CW1, and NL than in GGAT-AACC and GGAT-GGAT diplotypes (Table 4, $\mathrm{p}<$ $0.05)$, while individuals with GGAT-AACC were significantly higher in NH than in AACC-AACC and GGAT-GGAT diplotypes (Table 4, $\mathrm{p}<0.05$ ).

\section{DISCUSSION}

The DIO2 gene is responsible for converting prohormone $\mathrm{T} 4$ to the active hormone T3 and plays important roles in the synthesis of $\mathrm{TH}$ and the process of development. To date, the full-length coding sequence of the DIO2 gene has been cloned in humans [15], chickens [16], mice [17], and bovines [18] to study its expression level and regulation of TH. In the present study, the coding sequences of the $\mathrm{DIO} 2$ gene in
Sichuan White geese (knob geese) and Landes geese (nonknob geese) were cloned and had the closest homology with avians, which indicated a similar function between geese and other avians.

Robinson et al [19] showed that the appearance phenotypes of animals were associated with their growth, reproduction and other biological functions. For instance, an advantage in the fertility of horned males was found compared to polled males [20]. The various combs and wattles in chickens were related to egg weight, thermoregulation and sperm motility [21-23]. In the present study, individuals with the presence of a knob had greater head dimensions than those with the absence of a knob, which indicated that the knob phenotype might influence the skeletal development of the head in geese. Our previous results showed that the $\mathrm{DIO} 2$ gene played a pivotal role in determining the knob phenotype in geese and that a nonsynonymous mutation was identified in knob geese [14]. In the present study, four polymorphic loci of the DIO2 gene were found by comparison analysis of coding sequences between Sichuan White geese and Landes geese, which were further genotyped in Tianfu meat geese and three genotypes in each locus were identified. The values of $\mathrm{Ho}, \mathrm{He}$, and $\mathrm{Ne}$ reflected the degree of genetic variation in the population, and PIC represented the genetic information content [24]. Therefore, based on the analysis of the population indices and PIC value (Supplementary Table S2), our results showed that polymorphic loci of the $\mathrm{DIO} 2$ gene were at an interme-

Table 4. Association analysis between haplotype/diplotype of the deiodinase 2 gene and the head dimensions in the population of non-knob geese

\begin{tabular}{|c|c|c|c|c|c|c|c|c|c|c|c|c|c|c|}
\hline Hap/Dip & L1 & BL2 & BW1 & BW2 & BW3 & SW1 & CW2 & cW3 & ML & CL & UBH1 & UBH2 & NL & NH \\
\hline GAT & 8 & $94 \pm 0.47$ & 8 & 4 & $40 \pm 0.10$ & 1 & 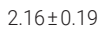 & 3 & 64 & I & 4 & 18 & .22 & $3 *$ \\
\hline$A C C$ & $2 \pm 0.38$ & 9 & $09 \pm 0.19 *$ & $2.65 \pm 0.15$ & $3 \pm 0.11$ & $16^{\star}$ & $2.19 \pm$ & 2.9 & $13.36 \pm 0.68$ & $7.99 \pm$ & $3.04 t$ & 0.17 & $2.92 \pm 0.19 *$ & 0.76 \\
\hline ACC-AACC & $6.32 \pm 0.38$ & 9 & $09 \pm 0.19 *$ & $2.65 \pm$ & 11 & $16^{*}$ & 2.19 & 2.91 & $13.36 \pm 0.68$ & $7.99 \pm$ & 3.0 & 1.7 & $2.92 \pm$ & $0.76 \pm 0.10$ \\
\hline S & $6.26 \pm$ & - & (1) & 12 & 09 & 17 & 2.1 & 2.95 & $13.12 \pm$ & 6 & 2.9 & 22 & 24 & $0.82 \pm 0.17 *$ \\
\hline GGAT-GGAT & $6.35 \pm 0.39$ & $6.94 \pm 0.47$ & $.00 \pm 0.18$ & $2.61 \pm 0.14$ & $2.40 \pm 0.10$ & $2.70 \pm 0.21$ & $2.16 \pm 0.19$ & $2.97 \pm 0.23$ & $13.27 \pm 0.64$ & $7.93 \pm 0.37$ & $3.03 \pm 0.24$ & $1.78 \pm 0.18$ & $2.82 \pm 0.22$ & $0.81 \pm 0.13$ \\
\hline
\end{tabular}

Centimeter $(\mathrm{cm})$ was used as the unit for length, height and width

BL1, beak length 1; BL2, beak length 2; BW1, beak width 1; BW2, beak width 2; BW3, beak width 3; CW1, cranial width 1; CW2, cranial width 2; CW3, cranial width 3; ML, maxillary length; CL, cranial length; $\mathrm{UBH} 1$, height of upper beak $1 ; \mathrm{UBH} 2$, height of upper beak $2 ; \mathrm{NL}$, nostril length; $\mathrm{NH}$, nostril height.

* Represented the statistical significance, $p<0.05$. 
diate level of genetic diversity. Furthermore, the dominant alleles at the g.419A $>$ G, g.533A $>$ G, g.725C $>$ A, and g.799C $>$ T loci were $G, G, A$, and T, respectively, and the frequencies of heterozygous genotypes of the four loci were higher than those of homozygous genotypes (Supplementary Table S2). Notably, the population of Tianfu meat geese was mainly used for breeding in growth and reproductive performance, while the knob phenotype was not the breeding target of this population. Therefore, the higher frequencies of heterozygous genotypes might be related to the artificial selection of the population, which is an important factor that can affect gene equilibrium in domestic animal populations [25]. Mentuccia et al [26] first identified a polymorphism of the DIO2 gene (Thr92Ala), and studies of Thr92Ala in the DIO2 gene were carried out in humans and mainly focused on its associations with diseases, such as Alzheimer's disease [27] and KashinBeck disease [28]. Another study also showed that the rs225017 polymorphism in the 3'UTR of the human DIO2 gene was associated with greater insulin resistance (IR) and interacted with the Thr92Ala polymorphism in the modulation of IR [29]. In the present study, the polymorphic loci of the DIO2 gene were significantly associated with BW1, BW2, NL, CW1, and $\mathrm{ML}$ in the population of non-knob geese. Moreover, the polymorphic loci of the $\mathrm{DIO} 2$ gene had a strong linkage association, and two haplotypes and three diplotypes were identified in the population of non-knob geese. Fallin et al [30] showed that studying the inheritance of haplotypes was more important than studying individual SNPs. In the present study, individuals with AACC haplotype/AACC-AACC diplotype were significantly higher in BW1, CW1 and NL than in GGAT haplotype/GGAT-AACC and GGAT-GGAT diplotypes. These results suggested that these polymorphic loci of the DIO2 gene might be used as molecular markers to select larger heads in the population of non-knob geese.

In conclusion, the head dimensions of geese were significantly different between knob and non-knob geese, and polymorphic loci of the DIO2 gene could be considered as molecular markers to select larger heads of geese in the population of non-knob geese.

\section{CONFLICT OF INTEREST}

We certify that there is no conflict of interest with any financial organization regarding the material discussed in the manuscript.

\section{FUNDING}

This work was funded by China Agricultural Research System of MOF and MARA, grant number CARS-42-4 and Key Technology Support Program of Sichuan Province, grant number 2021YFYZ0014.

\section{REFERENCES}

1. Bianco AC, da Conceicao RR. The deiodinase trio and thyroid hormone signaling. In: Plateroti M, Samarut J, editors. Thyroid hormone nuclear receptor. Methods Mol Biol 2018;1801: 67-83. https://doi.org/10.1007/978-1-4939-7902-8_8

2. Marsili A, Aguayo-Mazzucato C, Chen T, et al. Mice with a targeted deletion of the type 2 deiodinase are insulin resistant and susceptible to diet induced obesity. PLoS One 2011;6: e20832. https://doi.org/10.1371/journal.pone.0020832

3. Waung JA, Bassett JH, Williams GR. Adult mice lacking the type 2 iodothyronine deiodinase have increased subchondral bone but normal articular cartilage. Thyroid 2015;25:26977. https://doi.org/10.1089/thy.2014.0476

4. Bassett JHD, Boyde A, Howell P, et al. Optimal bone strength and mineralization requires the type 2 iodothyronine deiodinase in osteoblasts. Proc Natl Acad Sci USA 2010;107:7604-9. https://doi.org/10.1073/pnas.0911346107

5. Meulenbelt I, Min JL, Bos S, et al. Identification of DIO2 as a new susceptibility locus for symptomatic osteoarthritis. Hum Mol Genet 2008;17:1867-75. https://doi.org/10.1093/ $\mathrm{hmg} / \mathrm{ddn} 082$

6. Guo TW, Zhang FC, Yang MS, et al. Positive association of the DIO2 (deiodinase type 2) gene with mental retardation in the iodine-deficient areas of China. J Med Genet 2004;41: 585-90. https://doi.org/10.1136/jmg.2004.019190

7. Zhang $\mathrm{K}$, Xi H, Wang X, et al. A family-based association study of DIO2 and children mental retardation in the Qinba region of China. J Hum Genet 2012;57:14-7. https://doi.org/ 10.1038/jhg.2011.121

8. Heemstra KA, Hoftijzer H, Deure WM, et al. The type 2 deiodinase Thr92Ala polymorphism is associated with increased bone turnover and decreased femoral neck bone mineral density. J Bone Miner Res 2010;25:1385-91. https://doi.org/ 10.1002/jbmr.27

9. Uter JC, Krämer UM, Schöls L, et al. Single nucleotide polymorphisms in thyroid hormone transporter genes MCT8, MCT10 and deiodinase DIO2 contribute to inter-individual variance of executive functions and personality traits. Ex Clin Endocrinol Diabetes 2020;128:573-81. https://doi.org/10. 1055/a-1065-1786

10.Leitch VD, Bassett JHD, Williams GR. Role of thyroid hormones in craniofacial development. Nat Rev Endocrinol 2020;16:147-64. https://doi.org/10.1038/s41574-019-0304-5

11.Bakker B, Bikker H, Vulsma T, Randamie JS, Wiedijk BM, De Vijlder JJ. Two decades of screening for congenital hypothyroidism in The Netherlands: TPO gene mutations in total iodide organification defects (an update). J Clin Endocrinol Metab 2000;85:3708-12. https://doi.org/10.1210/jcem.85.10. 6878

12. Macchia PE, Lapi P, Krude H, et al. PAX8 mutations associated with congenital hypothyroidism caused by thyroid 
dysgenesis. Nat Genet 1998;19:83-6. https://doi.org/10.1038/ ng0598-83

13.Ji W, Hou lE, Yuan $\mathrm{X}$, et al. Identifying molecular pathways and candidate genes associated with knob traits by transcriptome analysis in the goose (Anser cygnoides). Sci Rep 2021;11: 11978. https://doi.org/10.1038/s41598-021-91269-1

14. Deng Y, Hu S, Luo C, et al. Integrative analysis of histomorphology, transcriptome and whole genome resequencing identified DIO2 gene as a crucial gene for the protuberant knob located on forehead in geese. BMC Genomics 2021;22: 487. https://doi.org/10.1186/s12864-021-07822-9

15. Croteau W, Davey JC, Galton VA, St Germain DL. Cloning of the mammalian type II iodothyronine deiodinase. A selenoprotein differentially expressed and regulated in human and rat brain and other tissues. J Clin Invest 1996;98:405-17. https://doi.org/10.1172/JCI118806

16. Gereben B, Bartha T, Tu HM, Harney JW, Rudas P, Larsen PR. Cloning and expression of the chicken type 2 iodothyronine 5'-deiodinase. J Biol Chem 1999;274:13768-76. https://doi. org/10.1074/jbc.274.20.13768

17.Song S, Sorimachi K, Adachi K, Oka T. Biochemical and molecular biological evidence for the presence of type II iodothyronine deiodinase in mouse mammary gland. Mol Cell Endocrinol 2000;160:173-81. https://doi.org/10.1016/ s0303-7207(99)00249-x

18. Connor EE, Laiakis EC, Fernandes VM, Williams JL, Capuco AV. Molecular cloning, expression and radiation hybrid mapping of the bovine deiodinase type II (DIO2) and deiodinase type III (DIO3) genes. Anim Genet 2005;36:240-3. https://doi.org/10.1111/j.1365-2052.2005.01282.x

19. Robinson MR, Pilkington JG, Clutton-Brock TH, Pemberton JM, Kruuk LE. Live fast, die young: trade-offs between fitness components and sexually antagonistic selection on weaponry in Soay sheep. Evolution 2006;60:2168-81. https://doi.org/ 10.1111/j.0014-3820.2006.tb01854.x

20.Constantinou A, Louca A, Mavrogenis AP. The effect of the gene for polledness on conception rate and litter size in the Damascus goat. Ann Genet Sel Anim 1981;13:111. https:// doi.org/10.1186/1297-9686-13-2-111

21.Al-Ramamneh DS, Makagon MM, Hester PY. The ability of White Leghorn hens with trimmed comb and wattles to ther- moregulate. Poult Sci 2016;95:1726-35. https://doi.org/10. 3382/ps/pew110

22. Wright $\mathrm{D}$, Boije $\mathrm{H}$, Meadows JRS, et al. Copy number variation in intron 1 of SOX 5 causes the Pea-comb phenotype in chickens. PloS Genet 2009;5:e1000512. https://doi.org/ 10.1371/journal.pgen.1000512

23. Imsland F, Feng C, Boije H, et al. The Rose-comb mutation in chickens constitutes a structural rearrangement causing both altered comb morphology and defective sperm motility. PloS Genet 2012;8:e1002775. https://doi.org/10.1371/journal. pgen.1002775

24.Zhu H, Zhang Y, Bai Y, et al. Relationship between SNPs of POU1F1 gene and litter size and growth traits in Shaanbei White cashmere goats. Animals 2019;9:114. https://doi.org/ 10.3390/ani9030114

25. Wang K, Cao Y, Rong Y, et al. A novel SNP in EIF2AK4 gene is associated with thermal tolerance traits in Chinese cattle. Animals 2019;9:375. https://doi.org/10.3390/ani9060375

26. Mentuccia D, Proietti-Pannunzi L, Tanner K, et al. Association between a novel variant of the human type 2 deiodinase gene Thr92Ala and insulin resistance: evidence of interaction with the Trp64Arg variant of the beta-3-adrenergic receptor. Diabetes 2002;51:880-3. https://doi.org/10.2337/diabetes. 51.3.880

27. McAninch EA, Rajan KB, Evans DA, et al. A common DIO2 polymorphism and alzheimer disease dementia in African and European Americans. J Clin Endocrinol Metab 2018; 103:1818-26. https://doi.org/10.1210/jc.2017-01196

28. Jin T, Wang L, He X, et al. Association between DIO2 polymorphism and the risk of Kashin-Beck disease in the Tibetan population. J Gene Med 2019;21:e3123. https://doi.org/10. 1002/jgm. 3123

29.Leiria LB, Dora JM, Wajner SM, Estivalet AA, Crispim D, Maia AL. The rs225017 polymorphism in the 3'UTR of the human DIO2 gene is associated with increased insulin resistance. PLoS One 2014;9:e103960. https://doi.org/10.1371/ journal.pone. 0103960

30. Fallin D, Cohen A, Essioux L, et al. Genetic analysis of case/ control data using estimated haplotype frequencies: application to APOE locus variation and Alzheimer's disease. Genome Res 2001;11:143-51. https://doi.org/10.1101/gr.148401 